\title{
Multi-Scale Simulations of Magnetic Reconnection Using Particle-in-Cell and Magnetohydrodynamics with Adaptive Mesh Refinement Technique*)
}

\author{
Tomoya OGAWA, Shunsuke USAMI ${ }^{1}$, Ritoku HORIUCHI ${ }^{1)}$, \\ Mitsue DEN $^{2)}$ and Kazuyuki YAMASHITA ${ }^{3)}$ \\ Kitasato University, Sagamihara 252-0373, Japan \\ ${ }^{1)}$ National Institute for Fusion Science, Toki 509-5292, Japan \\ 2) National Institute of Information and Communications Technology, Koganei 184-8795, Japan \\ 3) University of Yamanashi, Kofu 400-8510, Japan
}

(Received 9 December 2015 / Accepted 27 April 2016)

\begin{abstract}
We performed magnetic reconnection simulations with our simulation code. Our code is a multi-scale threedimensional plasma simulation code in which Particle-in-Cell (PIC) method is used in a restricted region and magnetohydrodynamics (MHD) simulation is performed in the rest of a simulation box. A hierarchical mesh is used in the MHD region, which is controlled by an adaptive mesh refinement (AMR) technique. Restriction of the PIC region and the adaptively controlled mesh realize treatment both of micro-scale particle dynamics and macro-scale structure with low computational costs. In this paper, we describe methods of our simulation and results of calculations.
\end{abstract}

(C) 2016 The Japan Society of Plasma Science and Nuclear Fusion Research

Keywords: multi-scale simulation, magnetic reconnection, PIC, MHD, AMR

DOI: $10.1585 /$ pfr. 11.2401096

\section{Introduction}

Magnetic reconnection is a multi-scale phenomenon, in which micro-scale particle dynamics, macro-scale fluid and magnetic structure are important. A magnetohydrodynamics (MHD) simulation is efficient for treating macroscale fluid, but it needs some kind of phenomenological resistivity model or extension of MHD. Different models of resistivity may lead to different results [1]. Extended MHD simulations are used (e.g. Otto 2001 [2]), but such simulations can only treat micro-scale effects partially. Particle simulations can treat particle dynamics directly, but the method needs high computational resources. In magnetic reconnection, micro-scale dynamics is important only near reconnection points, while micro-scale dynamics is not important far from reconnection points. Usami et al. developed a multi-scale simulation code [3-5]. They use Particle-in-Cell (PIC) method in a restricted region and perform MHD simulation in other regions. The multi-scale simulation code achieves the treatment of both micro-scale particle dynamics and macro-scale structure with lower computational costs than pure PIC simulations. Ogawa et al. extended their simulation box by connecting to a hierarchical mesh [6]. The hierarchical mesh is controlled by an adaptive mesh refinement (AMR) technique. It can extend a simulation box with a very small increase

author's e-mail: ogawa@kitasato-u.ac.jp

*) This article is based on the presentation at the 25th International Toki Conference (ITC25). in computational costs.

In this paper, we applied the simulation code to magnetic reconnection and show results below.

\section{Method}

A computational box is divided into three domains: a PIC domain, an interface domain, and an MHD domain. The latter two domains are separated into upper and lower regions by the centered PIC domain. A regular mesh is used in the PIC and the interface domains, and in the inner region of the MHD domain. A hierarchical mesh is used in the outer region of the MHD domain. Figures 1 and 2 show the structure of domains and meshes of two calculations.

PIC simulation is calculated in the PIC and the interface domains. MHD simulation is calculated in the interface and the MHD domains. In the interface domain, both PIC and MHD simulations are calculated simultaneously and exchange their information as below. Two sets of fluid quantities are derived by PIC and MHD algorithms: $Q_{\text {PIC }}$ and $Q_{\mathrm{MHD}}$, respectively. Figure 3 shows time developing procedure in the interface domain. The upper arrow represents time step in MHD and the lower arrow represents time step in PIC. At $t=t_{\mathrm{i}}, Q_{\mathrm{PIC}}$ is derived by averaging PIC fluid quantities over several steps and we obtain mixed quantities as

$$
Q_{\text {mix }}=\alpha Q_{\mathrm{MHD}}+(1-\alpha) Q_{\mathrm{PIC}},
$$

where the interconnection function $\alpha$ is within $0 \leq \alpha \leq 1$ 


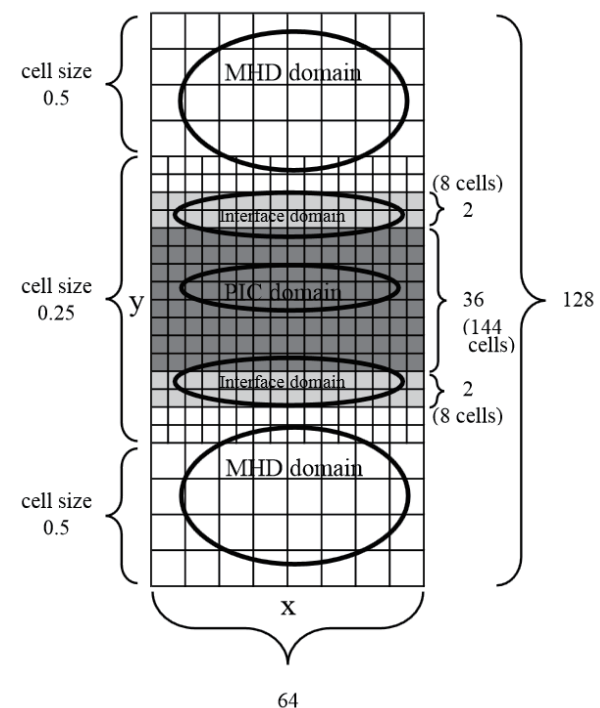

Fig. 1 Illustration of domains and mesh of calculation 1. Side lengths of a simulation box are $64 \times 128 \times 1$. Note that cell sizes drawn in the figure are not real. Actual cell sizes are 0.25 and 0.5 as described in left side of the figure. The maximum resolution corresponds to a regular $256 \times 512 \times 4$ mesh.

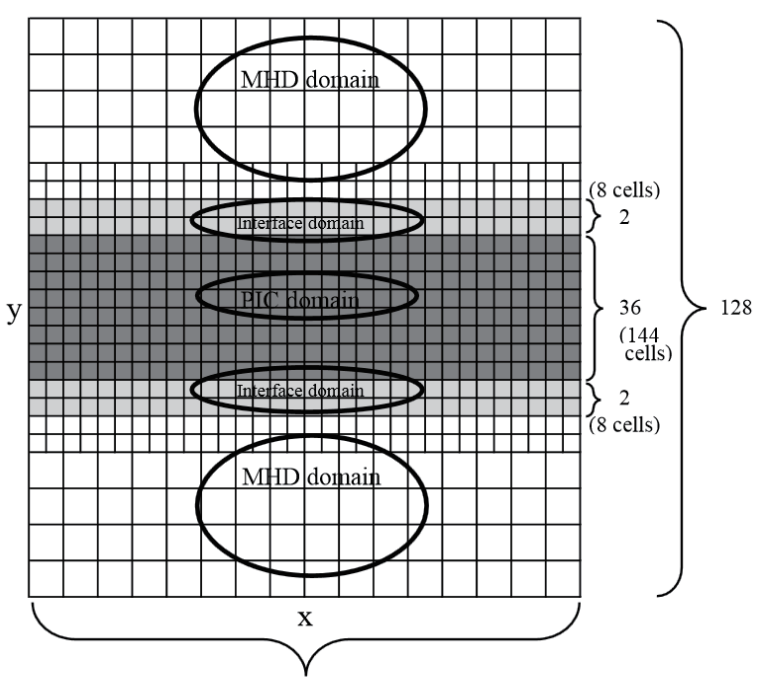

128

Fig. 2 Illustration of domains and mesh of calculation 2. Side lengths of a simulation box are $128 \times 128 \times 1$. Cell sizes are the same as in Fig. 1. The maximum resolution corresponds to a regular $512 \times 512 \times 4$ mesh.

and takes a large value near the MHD domain and a small value near the PIC domain. MHD quantities are replaced by the $Q_{\text {mix }}$ and the MHD time is advanced from $t_{\mathrm{i}}$ to $t_{\mathrm{i}}+$ $\Delta t_{\mathrm{MHD}}$, where $\Delta t_{\mathrm{MHD}}$ is a time step for the MHD scheme. The PIC macroscopic quantities such as the electric and magnetic fields, the density, and the pressure are replaced by the $Q_{\text {mix }}$. PIC particles are re-distributed at every step

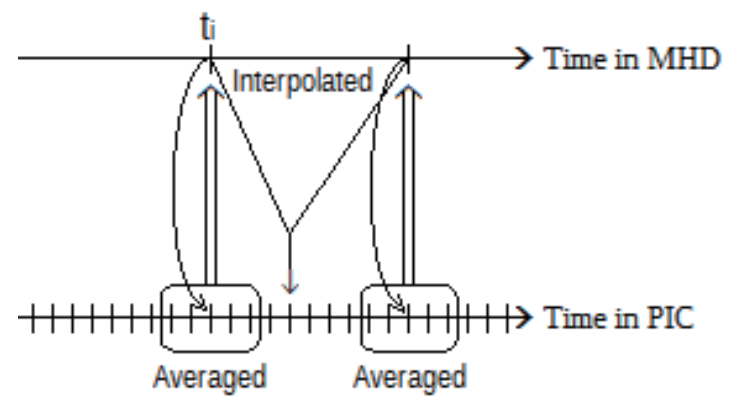

Fig. 3 Time developing procedure in the interface domain. The upper and lower rightward arrows are time in MHD and PIC schemes, respectively. Scales on these arrows represent time steps for each scheme.

based on the $Q_{\text {mix }}$ assuming a Maxwell distribution. At $t_{\mathrm{i}}<$ $t<t_{\mathrm{i}}+\Delta t_{\mathrm{MHD}}$, an interpolation of $Q_{\mathrm{MHD}}\left(t_{\mathrm{i}}\right)$ and $Q_{\mathrm{MHD}}\left(t_{i}+\right.$ $\Delta t_{\mathrm{MHD}}$ ) is used as $Q_{\mathrm{MHD}}$ in equation (1). See Usami et al. for details [3-5].

The hierarchical mesh is controlled by an AMR technique. The constitution unit of the mesh is "block" which consists of $2^{3}$ cells with physical quantities, pointers to six neighbors and eight children, and some additional quantities that are needed for control of the mesh structure or models. The blocks are connected to each other and construct a mesh structure as a Fully Threaded Tree [7]. The hierarchical mesh has cell sizes of the box size divided by $2^{l}$, where $l$ is a natural number. The initial mesh should be set manually. After starting calculation, mesh structure is decided by criteria for choosing refine or unrefine. We use expression (2) to detect shock wave and expression (3) to detect contact discontinuity:

$$
\begin{aligned}
& \frac{\left|P_{i}-P_{j}\right|}{\min \left(P_{i}, P_{j}\right)}>\varepsilon_{\mathrm{s}} \quad \text { and } \quad u_{i}>u_{j}, \\
& \frac{\left|P_{i}-P_{j}\right|}{\min \left(P_{i}, P_{j}\right)}<\varepsilon_{\mathrm{s}} \quad \text { and } \quad \frac{\left|\rho_{i}-\rho_{j}\right|}{\min \left(\rho_{i}, \rho_{j}\right)}>\varepsilon_{\mathrm{c}},
\end{aligned}
$$

where $P, u$, and $\rho$ are the pressure, the velocity component in a direction, and the density, respectively, $\varepsilon_{\mathrm{s}}$ and $\varepsilon_{\mathrm{c}}$ are the thresholds for shock wave and contact discontinuity, respectively, and subscriptions $i$ and $j$ denote the cells having smaller and larger coordinate value, respectively [7]. The values of $\varepsilon_{\mathrm{s}}$ and $\varepsilon_{\mathrm{c}}$ should be decided empirically. We set $\varepsilon_{\mathrm{s}}=0.5$ and $\varepsilon_{\mathrm{c}}=0.1$ in present calculations. If expression (2) or (3) is satisfied for a pair of cells in a block, then the block is divided into eight children having onehalf the length of their parent. In addition, we also divide surrounding blocks of the block that has been divided by the criteria.

\section{Models}

The MHD basic equations are 


$$
\left\{\begin{array}{l}
\frac{\partial \rho}{\partial t}=-\nabla \cdot(\rho \boldsymbol{u}) \\
\frac{\partial(\rho \boldsymbol{u})}{\partial t}=-\nabla \cdot(\rho \boldsymbol{u} \boldsymbol{u}-\boldsymbol{B B})-\nabla\left(P+\frac{B^{2}}{2}\right) \\
\frac{\partial \boldsymbol{B}}{\partial t}=\nabla \times(\boldsymbol{u} \times \boldsymbol{B}) \\
\frac{\partial e}{\partial t}=-\nabla \cdot\left[\left(e+P+\frac{\boldsymbol{B}^{2}}{2}\right) \boldsymbol{u}-\boldsymbol{B}(\boldsymbol{u} \cdot \boldsymbol{B})\right],
\end{array}\right.
$$

where $\rho, \boldsymbol{u}, \boldsymbol{B}, P$, and $e$ are the mass density, the fluid velocity, the magnetic field, the pressure, and the energy density, respectively. The governing equations in PIC algorithm are

$$
\left\{\begin{array}{l}
\frac{\partial \boldsymbol{B}}{\partial t}=-\nabla \times \boldsymbol{E} \\
\frac{\partial \boldsymbol{E}}{\partial t}=\nabla \times \boldsymbol{B}-C_{J} \boldsymbol{J} \\
\nabla \cdot \boldsymbol{B}=0 \\
\nabla \cdot \boldsymbol{E}=C_{J} \rho_{q} \\
\frac{\mathrm{d}\left(\gamma_{k} v_{k}\right)}{\mathrm{d} t}=\frac{q_{k}}{m_{k}}\left(\boldsymbol{E}+\boldsymbol{v}_{k} \times \boldsymbol{B}\right) \\
\frac{\mathrm{d} \boldsymbol{x}_{k}}{\mathrm{~d} t}=\boldsymbol{v}_{k},
\end{array}\right.
$$

where $\boldsymbol{E}, \boldsymbol{J}$, and $\rho_{q}$ are the electric field, the current density, and the charge density, respectively, $\gamma_{k}, \boldsymbol{v}_{k}, q_{k}, m_{k}$, and $\boldsymbol{x}_{k}$ are the Lorenz factor, the velocity, the charge, the mass, and the position of the $k$-th particle, respectively, $\boldsymbol{J}$ and $\rho_{q}$ are given by

$$
\left\{\begin{array}{l}
\boldsymbol{J}=\sum_{k} q_{k} \boldsymbol{v}_{k} S\left(\boldsymbol{x}-\boldsymbol{x}_{k}\right) \\
\rho_{q}=\sum_{k} q_{k} S\left(\boldsymbol{x}-\boldsymbol{x}_{k}\right),
\end{array}\right.
$$

where $S$ is a form function of super-particles, and the constant $C_{J}$ is defined as

$$
C_{J}=\left(\frac{\omega_{\mathrm{pe}}}{\omega_{\mathrm{ce}}}\right)^{2} \cdot n_{0}^{-1}\left(\frac{c}{\omega_{\mathrm{ce}}}\right)^{-3},
$$

where $\omega_{\mathrm{pe}}$ is the electron plasma frequency, $\omega_{\mathrm{ce}}$ is the electron gyrofrequency, $n_{0}$ is the reference value of particle number density, and $c$ is the light speed. Normalization constants of the MHD and PIC algorithms are different, thus we perform unit transformation. See Usami et al. (2013) for details [4].

Figures 1 and 2 show the structure of domains and meshes in two calculations: calculation 1 with the box size of $64 \times 128 \times 1$ and calculation 2 with that of $128 \times 128 \times 1$ (length is normalized by $c / \omega_{\text {ce }}$ ). The two calculations differ only in box sizes. Note that cell sizes drawn in these figures are not real. The cell sizes are 0.5 or 0.25 , thus the maximum resolution of calculations 1 and 2 corresponds to regular $256 \times 512 \times 4$ and $512 \times 512 \times 4$ meshes, respectively. In both calculations, the PIC domain is located on $46<y<82$, the interface domain is located on

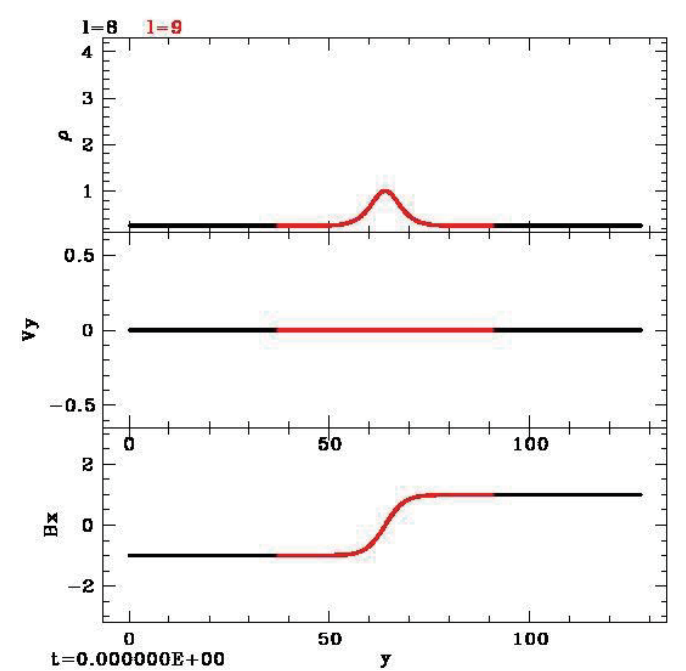

Fig. 4 Plots of density (top), $y$-component of velocity (middle), and $x$-component of magnetic field (bottom) for initial condition on a line with $x=$ constant and $z=$ constant. Color represents cell size in initial mesh structure; black and red indicate cell sizes of 0.5 and 0.25 , respectively.

$44<y<46$ and $82<y<84$, and the MHD domain is located on outer regions. Cell sizes of the PIC and the interface domains and the inner part of the MHD domain are always 0.25 . Cell sizes of the outer region of the MHD domain are 0.25 or 0.5 . In initial mesh, cell size is 0.25 in $36.75<y<91.25$ and is 0.5 in other regions. In outer region, there are no shock waves and no contact discontinuities in the present model, thus the mesh shows few change in its structure.

Initial condition is given at $t=0$ as

$$
\left\{\begin{array}{l}
\rho=\rho_{0}\left[a+(1-a)\left(1-\tanh ^{2} \frac{y-y_{\mathrm{c}}}{W}\right)\right] \\
\boldsymbol{u}=\mathbf{0} \\
P=P_{0}\left[a+(1-a)\left(1-\tanh ^{2} \frac{y-y_{\mathrm{c}}}{W}\right)\right] \\
\boldsymbol{B}=\boldsymbol{B}_{\mathbf{0}} \tanh \frac{y-y_{\mathrm{c}}}{W}
\end{array}\right.
$$

where the density and the pressure on $y=y_{\mathrm{c}}$ are $\rho_{0}=1$ and $P_{0}=2 / 3$, respectively, the constant for magnetic field is $\boldsymbol{B}_{\mathbf{0}}=(1,0,0)$ (magnetic field is normalized by $c \omega_{\text {ce }} m_{\mathrm{e}} / q_{\mathrm{e}}$, where $m_{\mathrm{e}}$ is the electron mass and $q_{\mathrm{e}}$ is the electron charge). The number ratio of background to foreground (Harris-equilibrated) particles is $a=0.25$, and the center position and the thickness (the length in $y$-direction) of a current sheet are $y_{\mathrm{c}}=64$ and $W=4.77$, respectively. Figure 4 shows plots of the initial condition together with the initial cell sizes.

We give electric field on $y$-boundary cells to drive plasma inflow as

$$
E_{z}=\left\{\begin{array}{cc}
\frac{E_{0}}{2}\left[1-\cos \frac{\pi t}{D}\right] & (t<D) \\
E_{0} & (t \geq D)
\end{array}\right.
$$


where the terminal strength of electric field is $E_{0}=-0.6$ and the increasing duration is $D=12.5$ (time is normalized by $c /\left(\omega_{\mathrm{ce}} v_{\mathrm{A}}\right)$, where $v_{\mathrm{A}}$ is the Alfvén speed). $x$ - and $z$ boundaries are periodic.

\section{Results}

Figures 5 and 6 show the results of calculation 1. Figure 5 displays color contours of density, $x$-component of velocity, $x$ - and $y$-components of magnetic field from left to right at $t=80$ on the upper panels and at $t=119$ on the lower panels. Figure 6 is plots of density, $y$-component
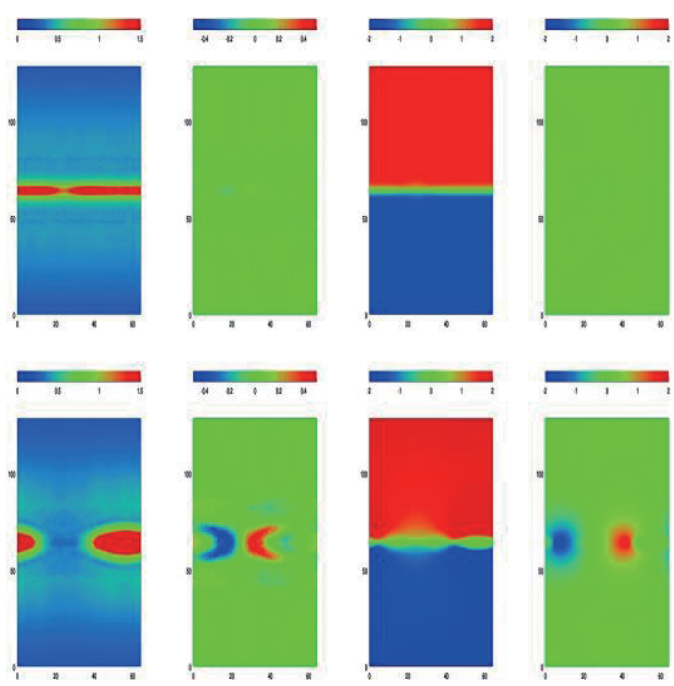

Fig. 5 Results of calculation 1. Color contours of density, $x$ component of velocity, $x$-component of magnetic field, and $y$-component of magnetic field (from left to right) on the plane of $z=0.625$ at $t=80$ (upper) and $t=119$ (lower).

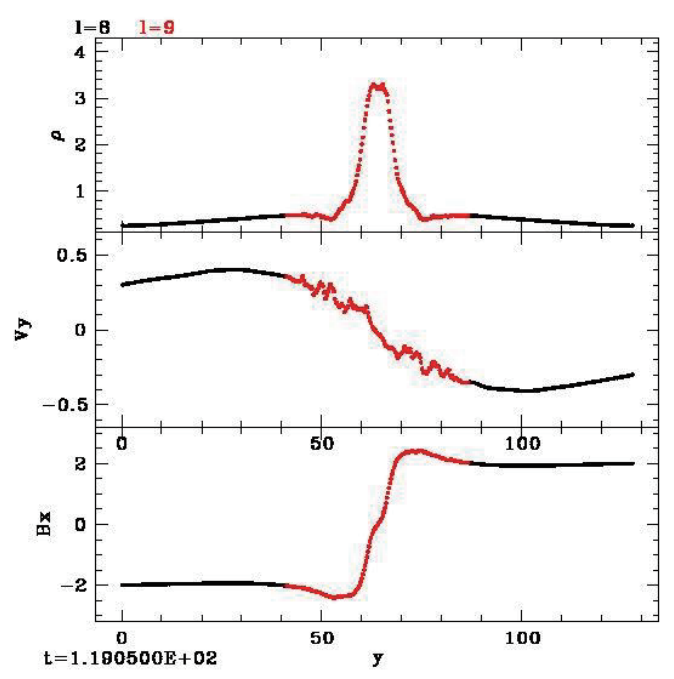

Fig. 6 Results of calculation 1. Plots of density (top), $y$ component of velocity (middle), and $x$-component of magnetic field (bottom) on the line of $x=32, z=0.625$ at $t=119$. Color has the same meaning in Fig. 4 . of velocity, and $x$-component of magnetic field from top to bottom at $t=119$. Figures 7 and 8 show the results of calculation 2. Figure 7 displays color contours of density on the left panels and $x$-component of velocity on the right panels at $t=96$ on the upper panels and at $t=119$ on the lower panels. Figure 8 is the same plots as Fig. 6 at $t=119$, however, this figure displays the results from calculation 2 . In both calculations, inflows run smoothly from upper and lower boundary to the centered PIC domain via the MHD domain with the hierarchical mesh and the interface domain, and then approach the center. Reconnection with single $x$-point occurs in calculation 1 . On the other hand, two $x$-points appear in the case of calculation 2. This is because the current sheet in calculation 2 is longer than that in calculation 1 . This result is consistent with $\mathrm{Ji} \&$
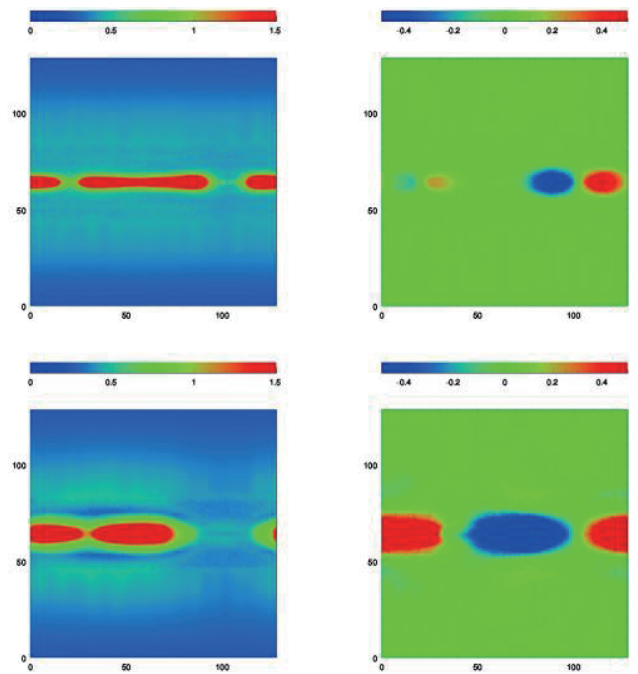

Fig. 7 Results of calculation 2. Color contours of density (left) and $x$-component of velocity (right) on the plane of $z=$ 0.625 at $t=96$ (upper) and $t=119$ (lower).

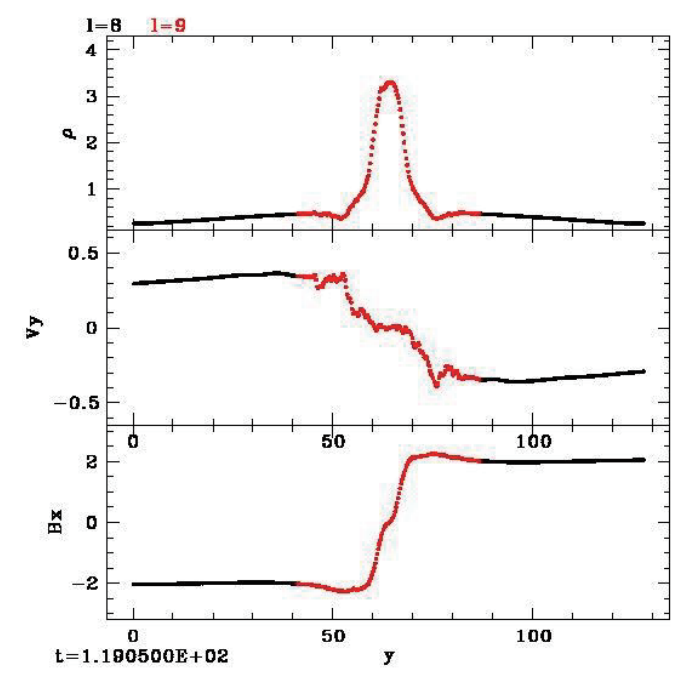

Fig. 8 The same plots as Fig. 6 but for calculation 2 on the line of $x=64, z=0.625$. 
Daughton (2011) qualitatively [8]. Fluctuations in the PIC domain are well suppressed in the interface domain.

We estimate computational gain by restriction of PIC region. The calculation 1 spent memory size of 105.2 GB and CPU time of 23.3 hours. For comparison, let us assume that the whole region is calculated by a pure PIC simulation. Such a calculation will need large memory and a long period of time. Furthermore, memory size and CPU time in PIC simulations are not proportional to the $y$-length of computational box because the number density of particles depends on the upstream direction. In the upstream far away from the current sheet, the plasma density is approximately uniform. Thus, we perform two smaller calculations, in which box sizes are $64 \times 40 \times 1$ and $64 \times 48 \times 1$ (grid numbers are $256 \times 160 \times 4$ and $256 \times 192 \times 4$ ), and then estimate costs in the case of a $64 \times 128 \times 1$ box $(256 \times 512 \times 4$ grids $)$ by assuming that the cost increment is proportional to the elongation of $y$-length. Spent memory and CPU time in the former calculation are 101.6 GB and $36565 \mathrm{sec}$. On the other hand, spent memory and CPU time in the latter calculation are $121.2 \mathrm{~GB}$ and $49522 \mathrm{sec}$. The box elongation of 8 in $y$-direction brings enhancements of memory size of $19.6 \mathrm{~GB}$ and CPU time of $12957 \mathrm{sec}$. Therefore, we can estimate computational costs in the case of $64 \times 128 \times 1$ as memory size of $317.2 \mathrm{~GB}$ and CPU time of 49.7 hours. This indicates that our method reduced memory usage to about one-third and CPU time to about one-half in comparison with a pure PIC calculation.

\section{Conclusion}

We applied our multi-scale three-dimensional plasma simulation code to magnetic reconnection. PIC region is restricted near the current sheet. Inflow from $y$-boundary has propagated smoothly to the PIC domain through the hierarchical mesh region and the interface domain. Magnetic reconnection with one or two $x$-points occurred on a current sheet. PIC noises have been well suppressed in the interface domain. By restricting the PIC region, we reduced memory usage to about one-third and CPU time to about one-half comparison with a pure PIC calculation. The outer hierarchical mesh will be able to extend a computational box with low cost enhancement. Thus, we can expect that future simulations with larger boxes will achieve higher computational gain. By this feature, we plan to connect our code with a global simulation involving a whole system, e.g., an Earth's magnetosphere simulation.

\section{Acknowledgements}

This work was supported by JSPS Grant-in-Aid for Scientific Research (B) 23340182 and the General Coordinated Research at the National Institute for Fusion Science (NIFS15KNTS039).

[1] M. Ugai, Phys. Fluids B 4, 2953 (1992).

[2] A. Otto, J. Geophys. Res. 106, 3751 (2001).

[3] S. Usami et al., Commun. In Comput. Phys. 11, 1006 (2012).

[4] S. Usami et al., Phys. Plasmas 20, 061208 (2013).

[5] S. Usami et al., J. Phys. Conf. Ser. 561, 012021 (2014).

[6] T. Ogawa et al., JPS Conf. Proc. 1, 016013 (2014).

[7] A.M. Khokhlov, J. Comput. Phys. 143, 519 (1998).

[8] H. Ji and W. Daughton, Phys. Plasmas 18, 111207 (2011). 